\title{
Development of Calculation Software for Traction Power Supply Capacity Based on Average Traffic Map
}

\author{
Zhongyan Qiao*, Jiachang Hu, Jinghui Yu, Xi Zhang, Shuo Ma \\ School of Electrical and Electronic Engineering, Shijiazhuang Tiedao University, Shijiazhuang, China
}

\section{Email address:}

1114881681@qq.com (Zhongyan Qiao)

${ }^{*}$ Corresponding author

\section{To cite this article:}

Zhongyan Qiao, Jiachang Hu, Jinghui Yu, Xi Zhang, Shuo Ma. Development of Calculation Software for Traction Power Supply Capacity Based on Average Traffic Map. Science Discovery. Vol. 7, No. 3, 2019, pp. 134-139. doi: 10.11648/j.sd.20190703.11

Received: May 2, 2019; Accepted: June 11, 2019; Published: June 15, 2019

\begin{abstract}
Accurately constructing the calculation model of the railway traction power supply load is the technical basis for the scientific selection of the traction transformer. In order to cope with the confusion caused by various traction transformer wiring forms and improve the efficiency of traction power supply calculation, based on the idea of average traffic diagram, the paper theoretically deduces a realistic traction power supply load calculation model and develops traction power supply. Capacity engineering calculation software. The front end of the software system adopts the friendly LabVIEW programming language and the related database access in the background, and the experiment proves that the software has the characteristics of accurate calculation, easy installation and operation.
\end{abstract}

Keywords: Traction Power Supply, Load Current Calculation, Traction Power Supply Capacity Calculation, LabVIEW

\section{基于平均运量法的牵引供电容量计算软件研制}

\author{
乔中彦”，胡家昌，于景慧，张茜，马硕 \\ 石家庄铁道大学电气与电子工程学院, 石家庄, 中国 \\ 邮箱 \\ 1114881681@qq.com (乔中彦)
}

\begin{abstract}
摘要: 精确构建铁路牵引供电负荷的计算模型是科学选配牵引变压器的技术基础。为应对各种牵引变压器接线形式带 来的困惑和提高牵引供电计算的效率，论文在基于平均运量图思想的基础上，理论推导一种贴近实际的牵引供电负荷 计算模型, 并开发出牵引供电容量工程计算软件。该软件系统前台采用界面友好的LabVIEW编程语言、后台采用关联 型数据库Access, 且实验证明该软件具有计算准确、易于安装和操作的特点。
\end{abstract}

关键字: 汼引供电, 负荷电流计算, 牵引供电容量计算, LabVIEW

\section{1. 引言}

在工程上, 科学选配铁路诨引变压器必须依靠牵引供 电负荷的计算模型, 并要求基于该计算模型的工程计算软 件能适应各种牵引变压器的接线形式。然而, 受行业属性
所限和多学科知识的影响, 国内只有少数研究单位和机构 研究牵引负荷计算模型和配套软件。

目前, 有关负荷计算软件大都为井下煤矿负荷计算和 电力系统负荷计算, 张朋介绍了其常用的需要系数法和二 项式系数法 [1]。另外, 赵乾创提出的曲线法具有一定创新 型, 但在实际应用中较为少见[2]。针对普速货运铁路牵引 
供电负荷计算的软件开发方面的研究较少。我国常规铁路 大多采用直供方式[3]。陈桁在文献中详细阐述了铁路牵引 供电负荷计算的两种常用方法: 平均运量法和运行图法 [4]。 平均运量法建立在概率论基础上的一种计算方法。这种算 法计算简单，但其计算结果有一定误差。其适用与符合分 布均匀场合 [5]。运行图法建立在列车运行图的基础, 其计 算过程比较复杂, 但结果较直观。

在铁路牵引供电负荷计算中, 欲实现其有关电流和容 量的精确计算十分困难, 实际工程中大多依靠工程经验或 简化相关计算。而负荷计算的准确性影响一系列电气设备 的选择及保护整定值的确定, 故在工程计算中能快速准确 并能减少人为失误的计算出负荷电流及变压器容量尤为 重要。

本软件研制拟采用LabVIEW软件作为开发平台, 以 Access为底层关系数据库, 建立以平均运量法为基础的普 速货运铁路牵引供电容量计算模型, 然后通过软件编程, 实现普速货运铁路牵引负荷电流及容量的相关计算。最后, 使用算例验证软件计算的精确性。

\section{2. 牵引供电容量工程算法}

本软件在进行牵引供电负荷电流计算时, 采用平均运 量法, 对现有公式进行改进, 使之更适于工程使用。算法 流程图见图1。

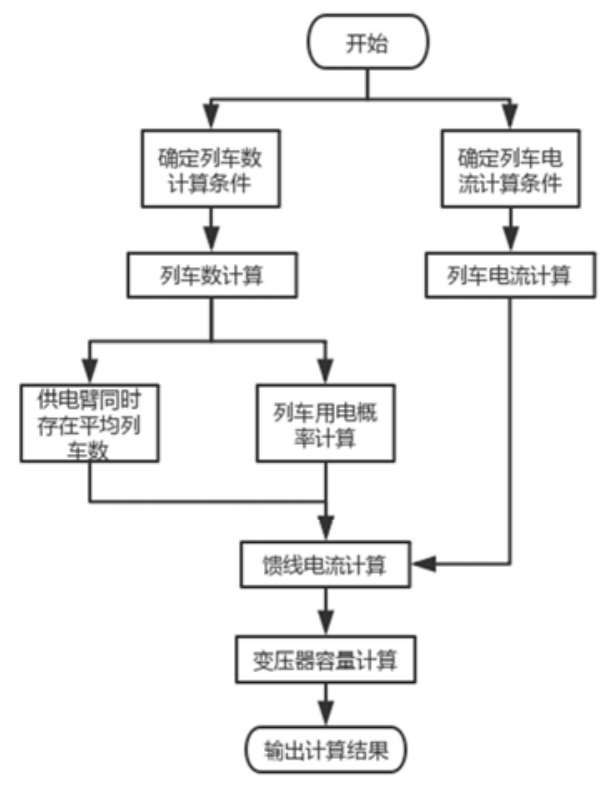

图1 工程算法流程图。

首先, 进行列车数计算, 由式:

$$
N=\frac{\Gamma_{\mathrm{J}} \times 10^{4}}{365 G \gamma_{\text {net }}}
$$

式中, $G$ 为列车牵引重量, 吨/列; $\gamma_{\text {net }}$ 为货物列车净 载系数; $\Gamma_{\mathrm{J}}$ 为线路货物年计算输送能力, $10^{4}$ 吨/年。本软 件研究针对普速货运铁路, 我国普速货运铁路主要为直接
供电方式, 且大部分是复线运行 $[6]$ 。故将 $\Gamma_{\mathrm{J}}$ 取值改进, 由 式:

$$
\Gamma_{\mathrm{J}}=1.38 \Gamma
$$

$\Gamma$ 为线路货物年需要输送能力, $10^{4}$ 吨/年。 其次, 进行列车电流计算时, 由式:

$$
I=\frac{60 \mathrm{~A}}{U t}=2.4 \frac{\mathrm{A}}{\mathrm{t}}
$$

式中, $\mathrm{A}$ 是列车通过供电分区的总能耗, $\mathrm{kVA} ; U$ 是 牵引网电压, 取 $25 \mathrm{kV} ; t$ 是列车通过供电分区的总的运行 时分, $\min$ 。

由于货运机车需要部分自用电取流, 且取流很小。故 计算列车电流时, 应加入该部分电流。张福生取自用电电 流为 $7 \mathrm{~A}[7]$ 。依据该公式可计算出列车平均电流。通过改 变参数和系数, 同样可计算出其他列车电流, 包括列车带 电平均电流计算、列车有效电流计算和列车带电有效电流 计算。

在列车数和列车电流计算完成之后, 进行馈线电流计 算, 并在计算时采用牵引计算的结果, 包括列车能耗、运 行时间和用电时间等参数, 然后进行母线电流计算。母线 电流计算式:

$$
I_{\mathrm{a} \varepsilon}=I_{\mathrm{a}} \sqrt{1+\frac{k_{\varepsilon \mathrm{g}}^{2}-2 p}{2 n p}}
$$

式中, $\mathrm{I}_{\mathrm{a}}$ 为馈线平均电流, $\mathrm{k}_{\varepsilon g}$ 由数据统计确定, $\mathrm{n}$ 为供 电分区数, $\mathrm{p}$ 为列车用电概率。

最后, 通过馈线电流参数可计算出不同变压器类型的 容量 $[8]$ 。例如三相 $Y N, d 11$ 接线牵引变压器计算容量式:

$$
\mathrm{S}_{\mathrm{c}}=\mathrm{K}_{\mathrm{t}} \mathrm{U}_{\mathrm{N}} \sqrt{4 \mathrm{I}_{\mathrm{a \varepsilon} 1}^{2}+\mathrm{I}_{\mathrm{a \varepsilon} 2}^{2}+2 \mathrm{I}_{\mathrm{a} 1} \mathrm{I}_{\mathrm{a} 2}} \quad(\mathrm{kVA})
$$

式中 $I_{\mathrm{a} \varepsilon 1} 、 I_{a 1}$ 为重负荷臂有效电流和平均电流, $\mathrm{A}$; $I_{\mathrm{a} \varepsilon 2} 、 I_{\mathrm{a} 2}$ 为轻负荷臂有效电流和平均电流, $\mathrm{A} ; U_{N}$ 为牵 引变电所牵引侧母线额定电压, $27.5 \mathrm{kV} ; K_{t}$ 为温度系数。

校核容量计算式:

$$
S_{\max }=\mathrm{K}_{\mathrm{t}} \mathrm{U}_{N}\left(2 \mathrm{I}_{1 \max }+0.65 \mathrm{I}_{a \varepsilon 2}\right) \quad(\mathrm{kVA})
$$

式中, $I_{1 \max }$ 为重负荷臂 1 最大电流; $I_{\mathrm{a} \varepsilon 2}$ 为轻负荷臂 2 有效电流。

\section{3. 软件开发环境及工程实现}

\section{1. 软件开发环境}

本软件前台开发采用LabVIEW软件工具, 其由美国 NI公司开发, 不仅可将程序界面图形化, 后台仍采用图形 化编程语言。LabVIEW编程不只编程语言简单, 还具有程 序调试和开发便捷以及强大的Internet功能等优点 [9]。同 时, 为方便后期增加新功能和修改程序, 软件结构采用模 
块化编程思想。后台采用Access数据库, 方便数据储存和 查询。

软件的主要功能模块包括两部分(见图2): 应用模块和 数据库模块。其中应用模块包括数据输入模块和计算模块, 数据库模块包括数据输入模块、数据库输出模块和报表模 块。

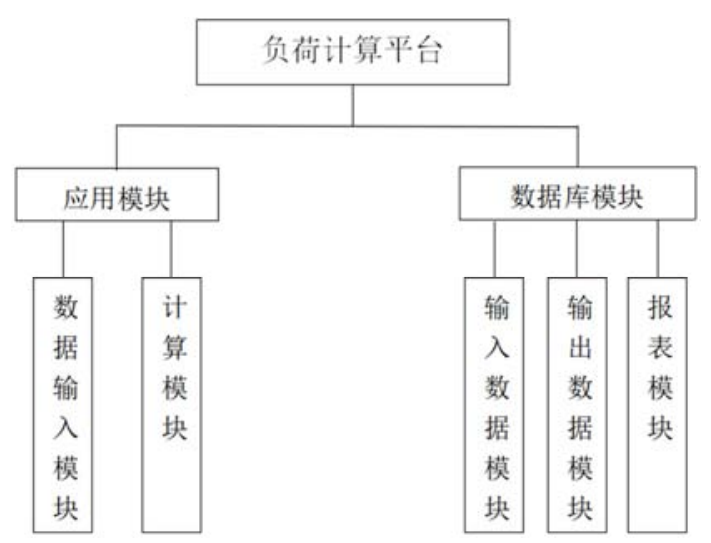

图2 软件功能模块。

\section{2. 容量计算软件工程实现}

软件操作流程为: 首先, 点击首界面的进入系统按钮, 牵引负荷计算输入界面被调用显示, 并且已通过程序设置 显示当前使用时间。根据界面控件标签输入相应的量, 点 击运行计算按钮, 通过程序使用LABSQL的工具包, 将计 算的输入数据和输出数据存储到ACCESS数据库中, 以便 查询使用记录等等。同时牵引负荷计算输出界面被调用显 示, 显示出相应的计算结果。点击制作报表按钮, 其将输 出数据显示在WORD模板中。点击查询记录按钮, 牵引负 荷计算查询界面被调用显示, 鼠标进入下拉列表控件时触 发事件, 显示出每次计算相应的时间, 对时间进行选择后, 点击查询按钮, 显示相应时间的输入数据和输出数据。每 个界面都有退出按钮, 点击退出按钮后, 退出相应界面。 软件流程图见图3-4, 具体程序见图5-6。

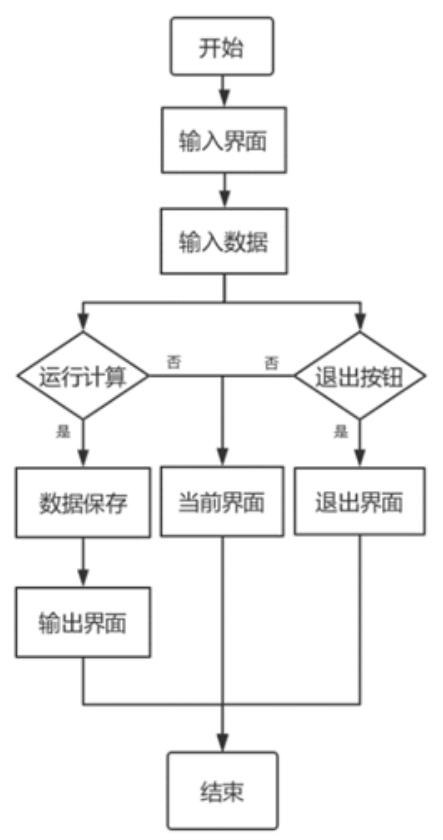

图3 输入界面流程图。

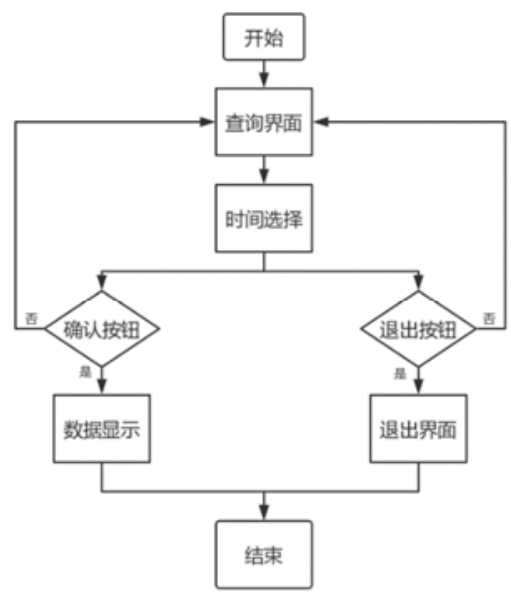

图4 查询程序流程图。

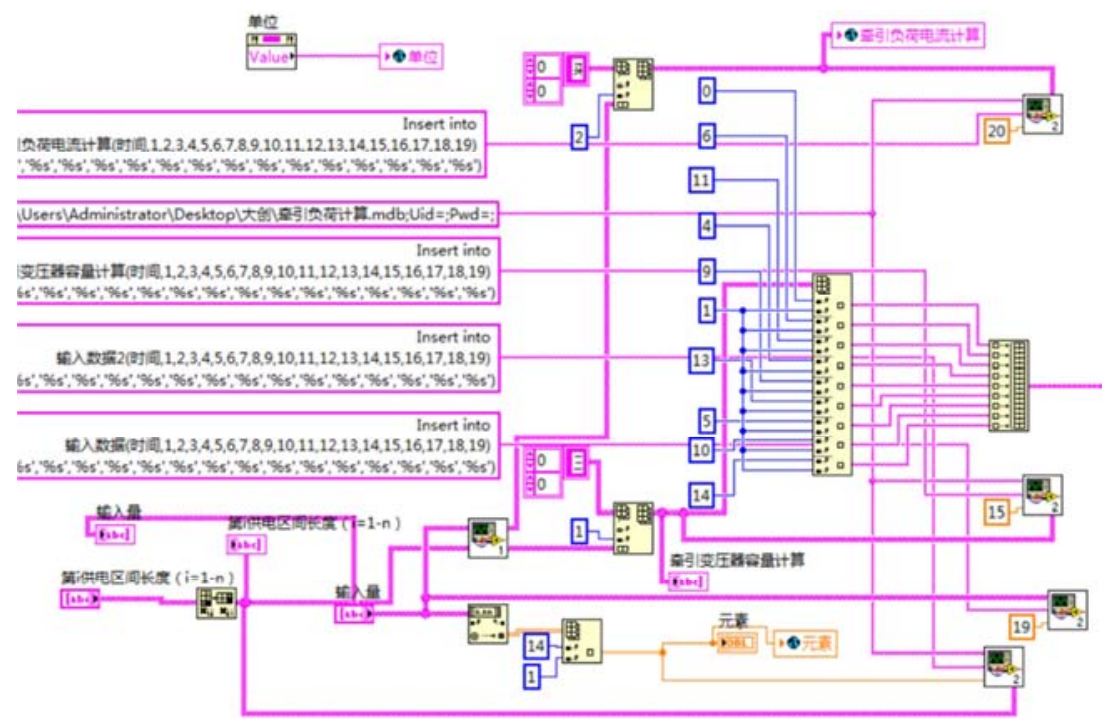

图5 算法编程图。 


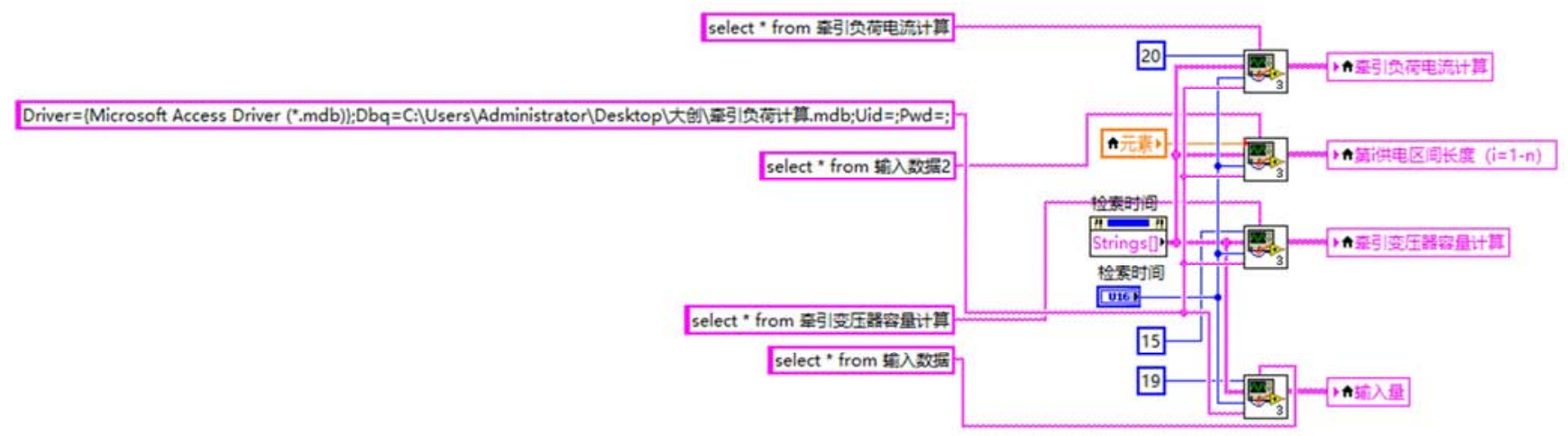

图6 查询数据程序图。

\section{4. 算例验证及分析}

基于上述模型, 对朔黄铁路某单线区段进行人工计算, 假定近期年运量 $\Gamma=2000$ 万吨/年, 诨引定数 $\mathrm{G}=2100$ 吨, $\gamma$ 静取 0.705 , 波动系数 $\mathrm{K} 1$ 取 1.2 , 储备系数 $\mathrm{K} 2$ 取 1.2 , 非平行 列车运行图区间通过能力 $\mathrm{N}$ 非 $=42$ 对/日。

牵引计算结果如下。
供电臂 $\alpha: \mathrm{n}=3, \sum \mathrm{A} 1=1724 \mathrm{kVAh}, \sum \mathrm{t}_{\mathrm{g} 1}=29.3 \mathrm{~min} ;$ 上 下行数据相同。

供电臂 $\beta: \mathrm{n}=3, \sum \mathrm{A} 2=2115 \mathrm{kVAh}, \sum \mathrm{t}_{\mathrm{g} 2}=28.3 \mathrm{~min} ;$ 上 下行数据相同。

接触网电压等级 $25 \mathrm{kV}$ 。选用三相YNd11接线牵引变压 器, 固定全备用。人工计算结果见表 1 。

表 1 算例人工计算结果。

\begin{tabular}{lll}
\hline 人工计算参数 & 供电臂 $\boldsymbol{\alpha}$ & 供电臂 $\boldsymbol{\beta}$ \\
\hline 列车对数 & 27 对/日 & \\
列车带电平均电流 & $141(\mathrm{~A})$ & $179(\mathrm{~A})$ \\
平均用电概率 & 0.55 & 0.53 \\
馈线平均电流 & $155(\mathrm{~A})$ & $190(\mathrm{~A})$ \\
馈线有效电流 & $199(\mathrm{~A})$ & $247(\mathrm{~A})$ \\
变压器计算容量 & $14485(\mathrm{kVA})$ & \\
变压器校核容量 & $34200(\mathrm{kVA})$ & \\
\hline
\end{tabular}

人工计算之后, 打开软件进行验证。首先登陆操作系 统(见图7), 进入系统后, 操作者按照系统输入界面提示输 入相应条件(见图8), 点击运行计算, 系统自动执行运算程

序。供电臂 $\alpha$ 计算结果计算结果(见图9), 供电臂 $\beta$ 计算结果 计算结果(见图9), 变压器容量计算结果(见图10)。由人工 计算与软件计算结果比较, 证明软件具有一定的精确性。

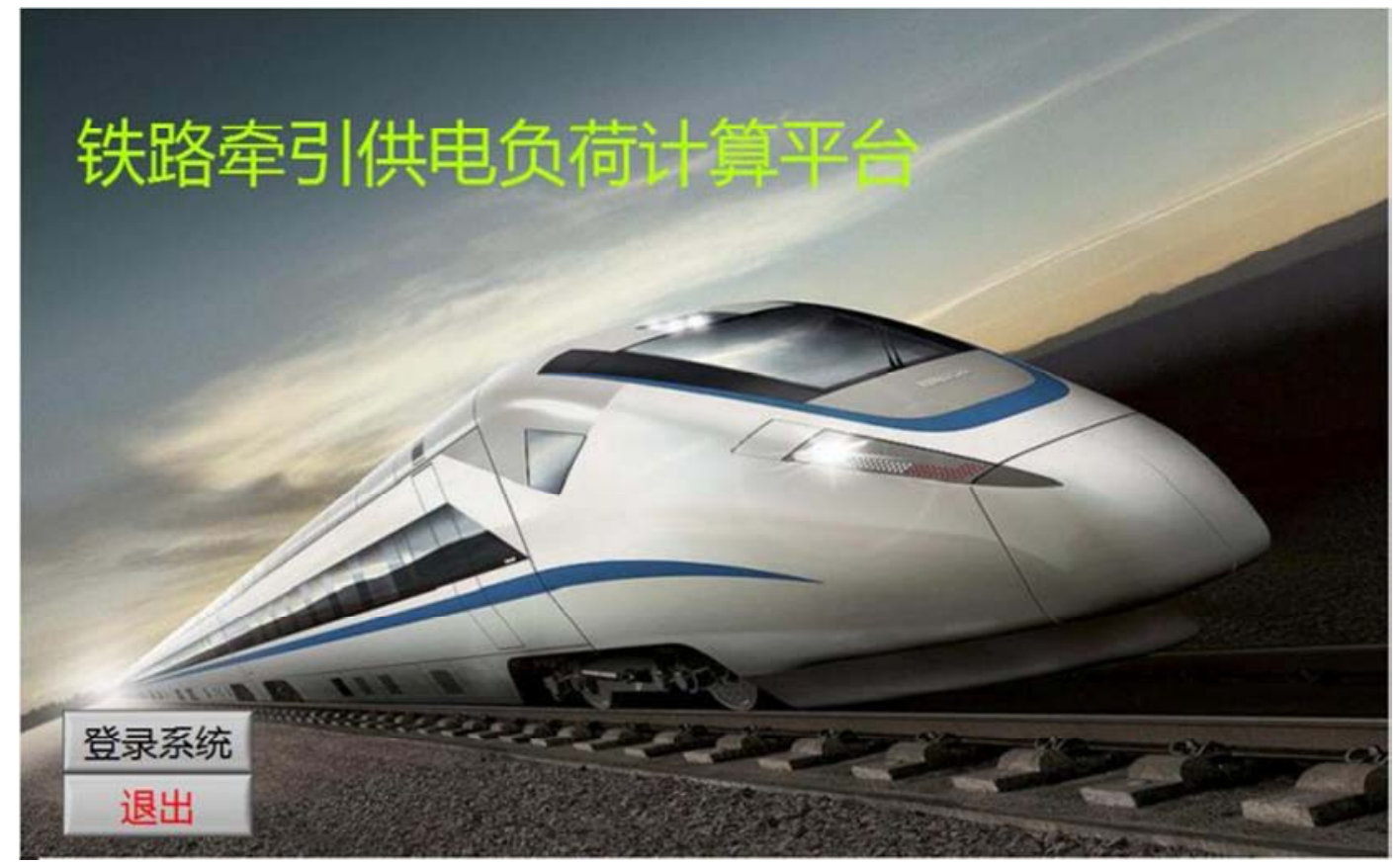

图7 进入系统界面。 


\section{石家庄铁道大学}

\begin{tabular}{|c|c|}
\hline & 供电臂 $\alpha$ \\
\hline 有效系数 & 货物列车净载重系数 \\
\hline 1.04 & 0.705 \\
\hline 牵引网电压(KV) & 区间带电走行时间(min) \\
\hline 25 & 29.3 \\
\hline 供电分区数 & 线路贷物年需要输送能力(万吨/年) \\
\hline 3 & 2000 \\
\hline 列车车引重量(吨/列) & 列车通过供电分区的总能耗(KVA*h) \\
\hline 2100 & 1724 \\
\hline 带电有效系数 & 非平行列车运行图区间通过能力(对/日) \\
\hline 1.04 & 42 \\
\hline 区间净走行时间(min) & 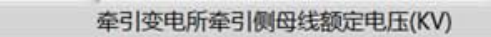 \\
\hline 25 & 27.5 \\
\hline 停车、会车时间(min) & 双线铁路追踪间隔连发列车时间 (min) \\
\hline 7 & 32 \\
\hline 供电分区总长度(KM) & 列车通过供电分区的总的运行时间(min) \\
\hline 500 & 29.3 \\
\hline
\end{tabular}

\begin{tabular}{|c|c|}
\hline & 供电臂 $\beta$ \\
\hline 有效系数 & 货物列车净载重系数 \\
\hline 1.04 & 0.705 \\
\hline 牵引网电压(KV) & 区间带电走行时间(min) \\
\hline 25 & 28.3 \\
\hline 供电分区数 & 线路货物年需要输送能力(万吨/年) \\
\hline 3 & 2000 \\
\hline 列车牵引重量(吨/列) & 列车通过供电分区的总能耗(KVA*h) \\
\hline 2100 & 2115 \\
\hline 带电有效系数 & 非平行列车运行图区间通过能力(对/日) \\
\hline 1.04 & 42 \\
\hline 区间净走行时间 (min) & 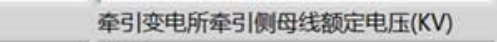 \\
\hline 25 & 27.5 \\
\hline 停车、会车时间(min) & 双线铁路追踪间隔连发列车时间 (min) \\
\hline 7 & 32 \\
\hline 供电分区总长度 (KM) & 列车通过供电分区的总的运行时间(min) \\
\hline 500 & 28.3 \\
\hline
\end{tabular}

单位

时间

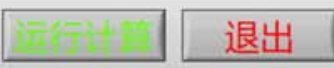

图8 输入界面。

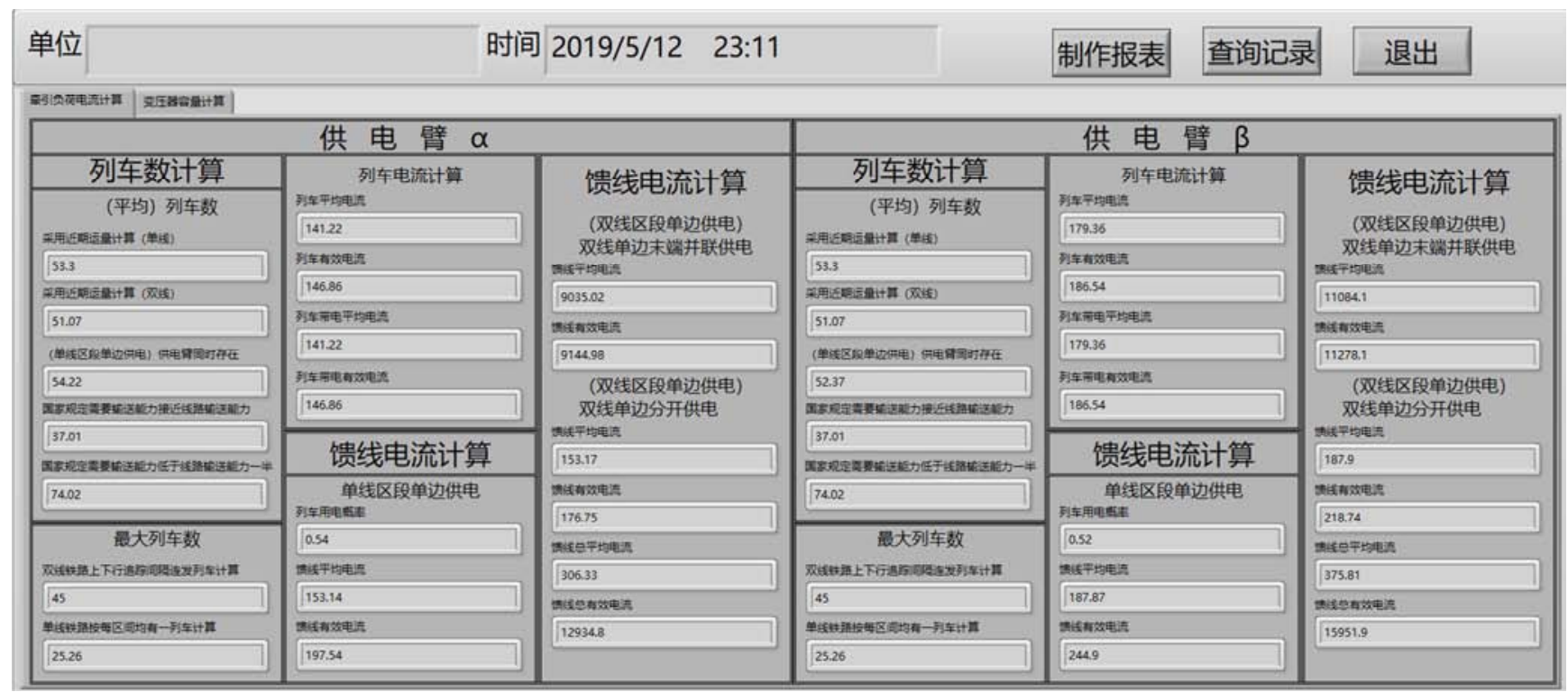

图9 计算结果。

\begin{tabular}{|c|c|c|}
\hline $\begin{array}{l}\text { 三相YN，d11接线 } \\
\text { 重引变压䎏计算蓉量 }\end{array}$ & 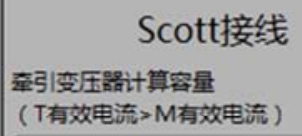 & 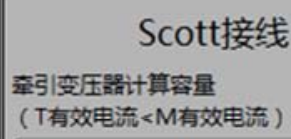 \\
\hline 14008 & 10129.9 & 11584.7 \\
\hline 虫引变压铝最大容量 & 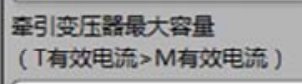 & 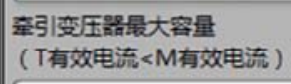 \\
\hline 51520.5 & 10334.8 & 11629.8 \\
\hline 金引|变压器校核察量 & 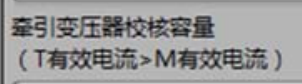 & $\begin{array}{l}\text { 晕引变压铝校核察量 } \\
\text { ( T有效电流<M有效电流) }\end{array}$ \\
\hline 34347 & 5167.37 & 5814.9 \\
\hline
\end{tabular}

图10 变压器容量计算结果。 
由人工计算与软件计算结果比较, 证明软件精确性较 强, 仍然存在一定误差。其误差原因主要有两点。其一, 由于线路本身损耗带有实时性，不易引入计算。铁路不同 区段的线路条件、运量和列车状况等不同造成铁路干线通 过能力不同, 从而对设备选择带来一定影响[10]。其二, 本软件研究采用平均运量法, 其建立在概率论基础上。计 算过程中, 某些中间数据本身带有一定误差, 故定会对计 算结果准确性带来影响。但总体来说, 其误差对牵引供电 容量选择影响可忽略。

\section{5. 结论}

本软件进行牵引供电容量计算时, 针对单线单边供电方 式、双线单边末端并联供电方式等不同供电方式, 采用同型 列车法, 得到的馈线电流, 最终实现三相 $\mathrm{YN}, \mathrm{d} 11$ 牵引变压器 和Sccot变压器的容量计算。软件工程性较强, 操作简单, 便 于工作人员在现场直接操作。且对硬件要求极低, 方便安装, 可直接在计算机上运行, 可强有力的帮助相关技术人员加大 工作效率，减少在设备选择和整定定值方面的偏差。

\section{致谢}

本文为中国大学生创新创业训练计划项目 《基于 $\mathrm{B} / \mathrm{S}$ 结构的朔黄铁路牵引供电负荷计算平台研究》的阶段性研 究成果之一，项目编号为201810107059。

\section{参考文献}

[1] 张朋.基于Visual Basic的供电负荷计算开发.科技创新与应 用。

[2] 赵乾钊. 牵引变电所馈线平均电流、有效电流新计算方法的 研究。

[3] 王梦兰.牵引供电系统馈线保护整定计算的研究。

[4] 陈桁, 胥伟.城市轨道交通牵引供电计算负荷与实际负荷差 异原因分析及应对措施.城市

[5] 万庆祝.基于牵引计算的牵引变电所馈线电流仿真计算。

[6] 林国松,马劲飞.基于分区所电抗的电气化铁路故障测距方 案轨道交通研究。

[7] 张福生.牵引供电系统. 北京交通大学出版社。

[8] 史天玉.基于馈线电流的牵引变压器容量计算。

[9] 邵林林.浅析LabVIEW编程语言。

[10] 孙晚华.基于运量结构的铁路干线通过能力计算方法研究。 